\title{
Automatic Thin Cap Fibroatheroma Detection Using Fusion of Intravascular Ultrasound and Virtual Histology Images
}

\author{
Zahra Rezaei 1,2, Ali Selamat 2,3,4,*, Arash Taki 5, Mohd Shafry Mohd Rahim 2, \\ Mohammed Rafiq Abdul Kadir 6, Hamido Fujita ${ }^{7}$, Enrique Videma Harera ${ }^{8,9}$, Ondrej Krejcar ${ }^{3}$ \\ 1 Department of Computer Engineering, Marvdasht Branch, Islamic Azad University, Marvdasht, Iran; \\ rzahra.rezaei@gmail.com \\ 2 School of Computing, Faculty of Engineering, Universiti Teknologi Malaysia (UTM) \& UTM-IRDA \\ Center of Excellence, 81310 UTM Johor Bahru, Johor, Malaysia; shafry@utm.my \\ 3 University of Hradec Kralove, Rokitanskeho 62, Hradec Kralove, 500 03, Czech Republic; \\ ondrej.krejcar@uhk.cz \\ 4 Malaysia Japan International Institute of Technology, Universiti Teknologi Malaysia (UTM), Kuala \\ 5 Technical University of Munich (TUM), Munich, Germany; taki@in.tum.de \\ 6 School of Biomedical, Faculty of Engineering, Universiti Teknologi Malaysia, 81310 UTM Johor Bahru, \\ Johor, Malaysia; rafiqkadir@utm.my \\ 7 Faculty of Software and Information Science, Iwate Prefectural University, Iwate 020-0693, Japan; \\ HFujita-799@acm.org \\ 8 Department of Computer Science and Artificial Intelligence, University of Granada. 18071 Granada, \\ Spain; viedma@decsai.ugr.es \\ 9 Department of Electrical and Computer Engineering, Faculty of Engineering, King Abdulaziz University, \\ Jeddah 21589, Saudi Arabia \\ * Correspondence: aselamat@utm.my
}

\begin{abstract}
Virtual Histology- Intravascular Ultrasound (VH-IVUS) image is an available method for visualizing plaque component to detect thin cap fibroatheroma. Nevertheless, this imaging modality has considerable limitations to extract the plaque component features and identifying the TCFA plaque. The aim of this paper is to improve the identification of TCFA using fusion of IVUS and VH-IVUS images. In order to generate the automatic technique for reducing the human interaction, a new method namely Active Contour based Plaque Border Detection (ACPB) is proposed. In order to perform the pixel wise classification, hybrid of K-means algorithm with Particle Swarm Optimization and Plaque based Minimum Euclidean Distance (KMPSO-PMED) method is presented to classify the plaque region as well. Moreover, to obtain more significant information of imaging modalities, fusion of two different images consisting of VH-IVUS and IVUS is performed. Therefore, geometric features are extracted from the plaque region and combine with IVUS features. Furthermore, different group of plaque features are divided by means of the histopathological studies. SVM classifiers is applied to detect the TCFA and non-TCFA plaques. The proposed method is applied on 566 in-vivo IVUS and their matching VH-IVUS images obtained from 9 patients. The best result of SVM illustrates the accuracy rates of $99.41 \%$ for classification of TCFA plaque. The results prove that the highest accuracy is achieved by integrated features of IVUS and VH-IVUS images.
\end{abstract}

Keywords: TCFA; VH-IVUS image; plaque border detection; active counter method

\section{Introduction}

Thin Cap Fibroatheroma (TCFA) plaque is particularly prone to rupture and causes cardiac events [1]. Invasive imaging modalities, including Intravascular Ultrasound (IVUS), Virtual Histology- 
Intravascular Ultrasound (VH-IVUS), and optical coherence tomography (OCT) have been presented for identifying the TCFA plaque [2]. Ultrasound imaging has been developed based on the high frequency sound waves to provide the images form body tissues and organs [3]. The first commercially available IVUS-based technology, namely VH-IVUS is presented by Nair, Kuban [4] for atherosclerotic plaque characterization. Proposed method has been implemented in Volcano (Rancho Cordova, CA) software to classify four tissue types consisting of dense calcium (DC), necrotic core (NC), fibrotic tissue (FI), fibro-fatty tissue (FF) [5]. Plaque characterization is required to accurately describe the vicinity of plaque component in contact with the lumen [6, 7]. However, the gold standard of plaque vulnerability is not available in clinical assessment $[8,9]$.

Some program-based studies have been investigated the diagnosis of the plaque type. In order to plaque type classification, a program was developed by the authors of Margolis, Vince [10]. Plaque type classification in IVUS images was performed by means of a decision tree. The authors of [11] attempted to classify the plaque by means of the simple threshold methods. But, their method was not consistent for segmentation of the highly mixed tissue types. The analysis of lesion morphology using baseline and follow-up VH-IVUS images has investigated by Kubo, Maehara [12]. However, proposed method only evaluated the plaque burden of non-culprit types. In [13] a program for assessment of atherosclerotic plaque morphology using IVUS/ VH-IVUS images has been developed. Developed software was capable to classify the TCFA and calcified TCFA plaques based on the phantom images. The authors of [14] classified VH-IVUS-defined images to examine the interobserver variability. The effect of different definitions for TCFA plaque were explored as well. Nevertheless, the presented algorithm was unable to recognize the thrombus. Moreover, the analyses were performed on plaques not on patients and provided the different results. Plaque type classification using the baseline images was presented by Zhang et al. [15]. Morphology structures of VHIVUS and IVUS images and biomarkers were presented to classify the TCFA and non-TCFA. Recently, Zhang et al. [1] addressed the prediction of TCFAs using the VH-IVUS data. However, TCFA is identified at a single-frame, while the length of VH-derived TCFA should be in at least three consecutive frames. Despite profound advance in atherosclerosis treatment, preventive and diagnostic tools should be developed to reduce the risk of sudden death $[8,9]$.

In our previous work [16], segmentation, geometric feature extraction, and machine learning based classification were proposed to diagnosis the TCFA plaque. Moreover, in our recently published paper [17], a new hybrid model of K-means with Particle Swarm Optimization (PSO) and a minimum Euclidean distance algorithm (KMPSO-mED) has been proposed to enhance the VHIVUS images segmentation. Furthermore, geometric and informative texture features were fused to provide a discriminative feature vector for TCFA detection. Different classifiers are applied to obtain the best accuracy result for classifying the plaque into TCFA and non-TCFA. In this paper, in order to generate an automatic technique of plaque border detection, a new method namely Active Contour based Plaque Border Detection (ACPB) is proposed. The delineated plaque area is presented to the pixel wise classification step of K-means PSO-Plaque Based Minimum Euclidean Distance model (KMPSOPMED) model for improving the accuracy of VH-IVUS image segmentation. Although, many new imaging modalities have been emerged, but ultrasound is applied as a popular imaging modality because it visualizes the most of tissues without damaging them [18]. Therefore, plaque features of IVUS and VH-IVUS images are fused to accurately diagnosis of the TCFA. Histopathological features are considered to present the different group of features for the classifier as well. This study is designed into five sections. The proposed methodology for feature extraction using VH-IVUS and IVUS images are presented in Section 2. Section 3 illustrates the classification results by means of different feature sets. The strong points and restrictions of the presented methods are discussed in Section 4 and the conclusion is presented in Section 5.

\section{Proposed Methodology}

The proposed approach consisting of plaque borders detection, segmentation, feature extraction using VH-IVUS and IVUS, data set labeling, and TCFA detection. In order to generate the automatic technique for reducing the human interaction, a new technique namely Morphological Lumen Based 
Initial Contour (MLIC) is proposed. The ACPB algorithm is then proposed to automatically detect the plaque borders. Plaque area is used by KMPSO-mED model to segment the VH-IVUS image. The Extracting Confluent Components (ECC), Determining NC layering (DNCL), and Plaque Burden Measurement (PBM) algorithms are employed to extract the geometric features of VH-IVUS images. Geometric features of IVUS and different group of VH-IVUS feature sets are fed to the classifier. Figure 1 illustrates the block diagram of the proposed approach.

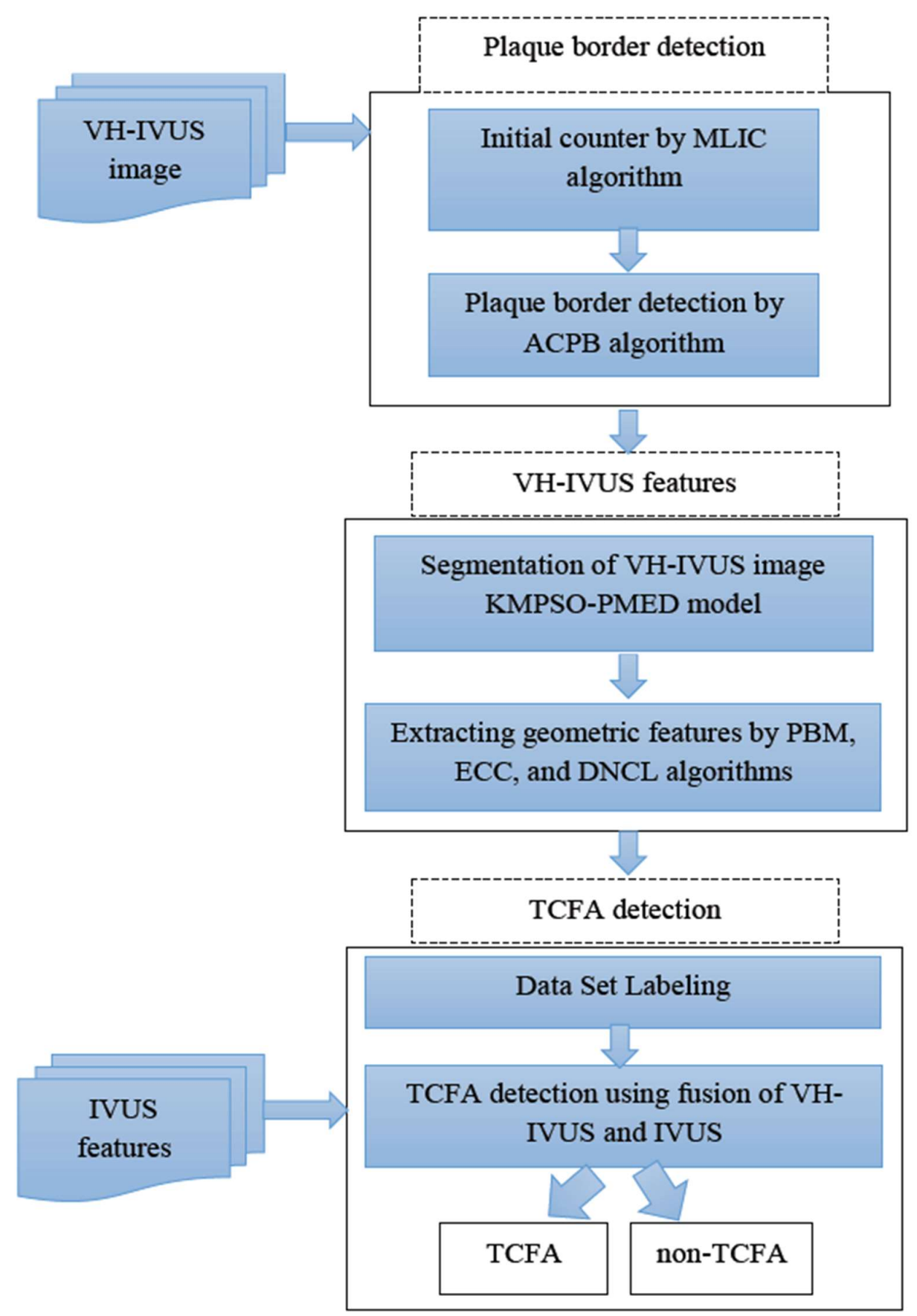

Figure 1. Proposed approach (MLIC: Morphological Lumen Based Initial Contour, ACPB: Active Contour based Plaque Border Detection KMPSO-PMED: hybrid of K-means PSO-Plaque Based Minimum Euclidean Distance model, ECC: Extracting Confluent Components, NCL: Determining NC layering, and PBM Plaque Burden Measurement). 


\subsection{Proposed Active Contour based Plaque Border Detection (ACPB) Algorithm}

\subsubsection{Morphological Lumen Based Initial Counter (MLIC)}

Initialization of contour in image is the essential step in level set methods. In order to generate the automatic technique for reducing the human interaction, a technique namely Morphological Lumen Based Initial Contour (MLIC) is proposed. Filtered VH-IVUS image is transformed to a new binary image namely $I P$ by means of the following equation. Where, $f(i, j)$ illustrates the intensity of VH-IVUS image in coordinates $(i, j)$.

$I_{P}=\left\{\begin{array}{l}1 f(i, j)>0 \\ 0 \text { otherwise }\end{array}\right.$

Lumen is a hole that is defined as an area of dark pixels surrounded by plaque region. To determine the vessel region, the zero values of the lumen area are converted into one, so vessel region namely $I_{V}$ can be detected by:

$I_{V}=\left\{\begin{array}{l}1 \quad f(i, j) \in \text { Lumen } \\ f(i, j) \text { otherwise }\end{array}\right.$

Lumen region $I_{L}$ is computed by subtracting the $I_{V}$ from $I_{P}$. After removing the small object in $I_{L}$, the biggest area is determined as lumen. By extracting the properties of the $I_{L}$, the smallest rectangle containing the lumen region of bounding box namely $B B x$ is calculated. This matrix specifies the upper-left corner of the $B B x$ and the width of the $B B x$ along each dimension.

$x_{1}=B B x(1), y_{1}=B B x(2)$

$x_{2}=B B x(3), y_{2}=B B x(4)$

Figure 2 illustrates the steps of MLIC algorithm. The $B B x$ counter is illustrate by red color around the lumen (Figure 2. (c)).

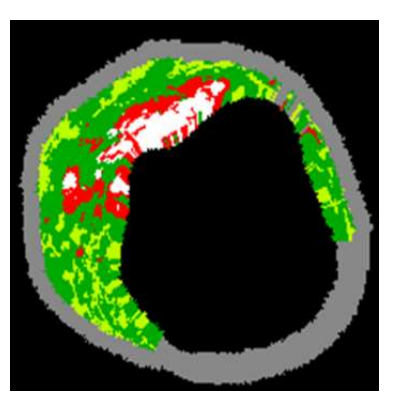

(a) VH-IVUS

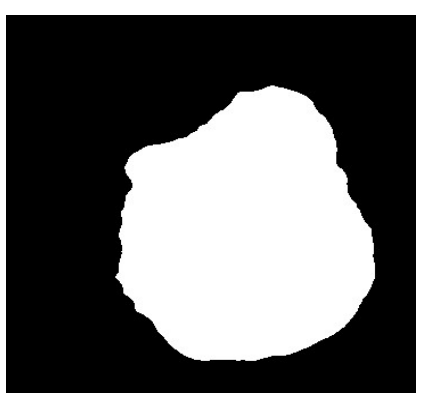

(b) Lumen

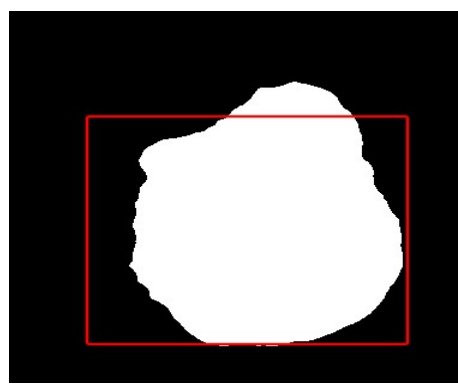

(c) Initial counter

Figure 2. Result of MLIC algorithm.

\subsubsection{Level Set Method}

The active contour method is presented to delineate the contour in region of interest (ROI) and detect the vessel and lumen borders [19]. Let surface $\varphi$ to represent the inside of $B B x$, where $\varphi(i, j)=-$ $1, i=x_{1}, \ldots, x_{1}+x_{2}$ and $j=y_{1}, \ldots, y_{1}+y_{2}$. However, the values outside the $B B x$ are positive. In order to enclose the curve within the surface, $\varphi$ is adjusted iteratively until convergence [20]. In VH-IVUS image, by considering the closed lumen, two borders should be detected. Therefore, counter initialization is very important. The detected plaque is considered as ROI that is called the plaque area (PA). The pixels of belong to the internal counter are considered as lumen border (LB). Figure 3 illustrates the result of different iterations for plaque border detection by ACPB algorithm. Figure 4 depicts the detected vessel and lumen borders for different VH-IVUS images using the proposed algorithm. 


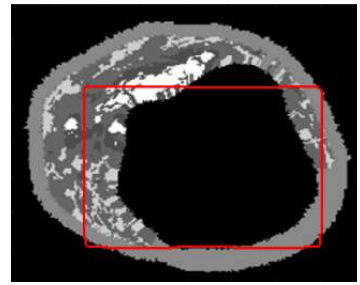

$1 \mathrm{st}$

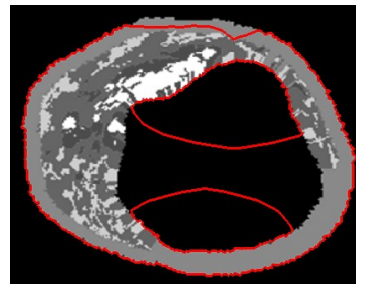

30th

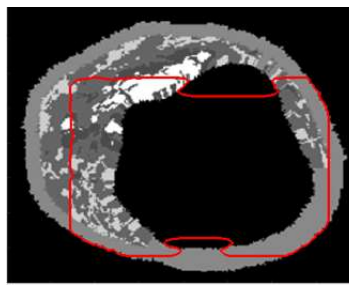

5th

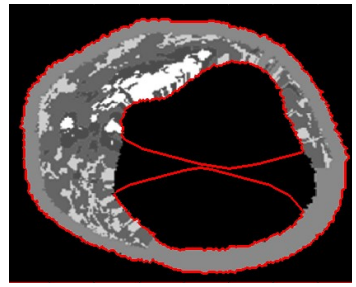

40th

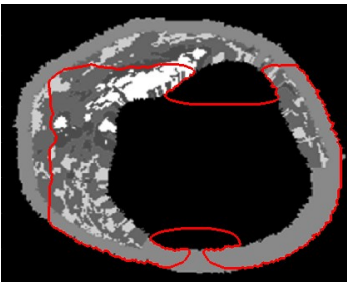

10th

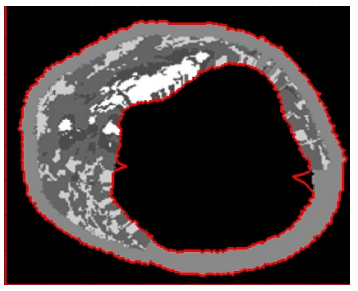

50th

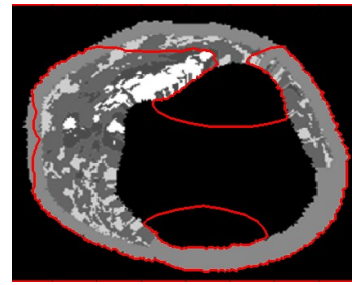

20th

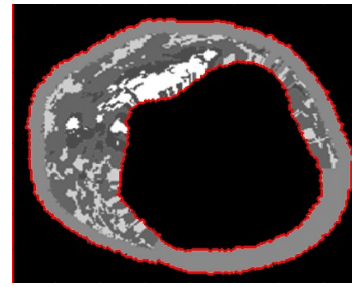

60th

Figure 3. Different iterations of ACPB algorithm.
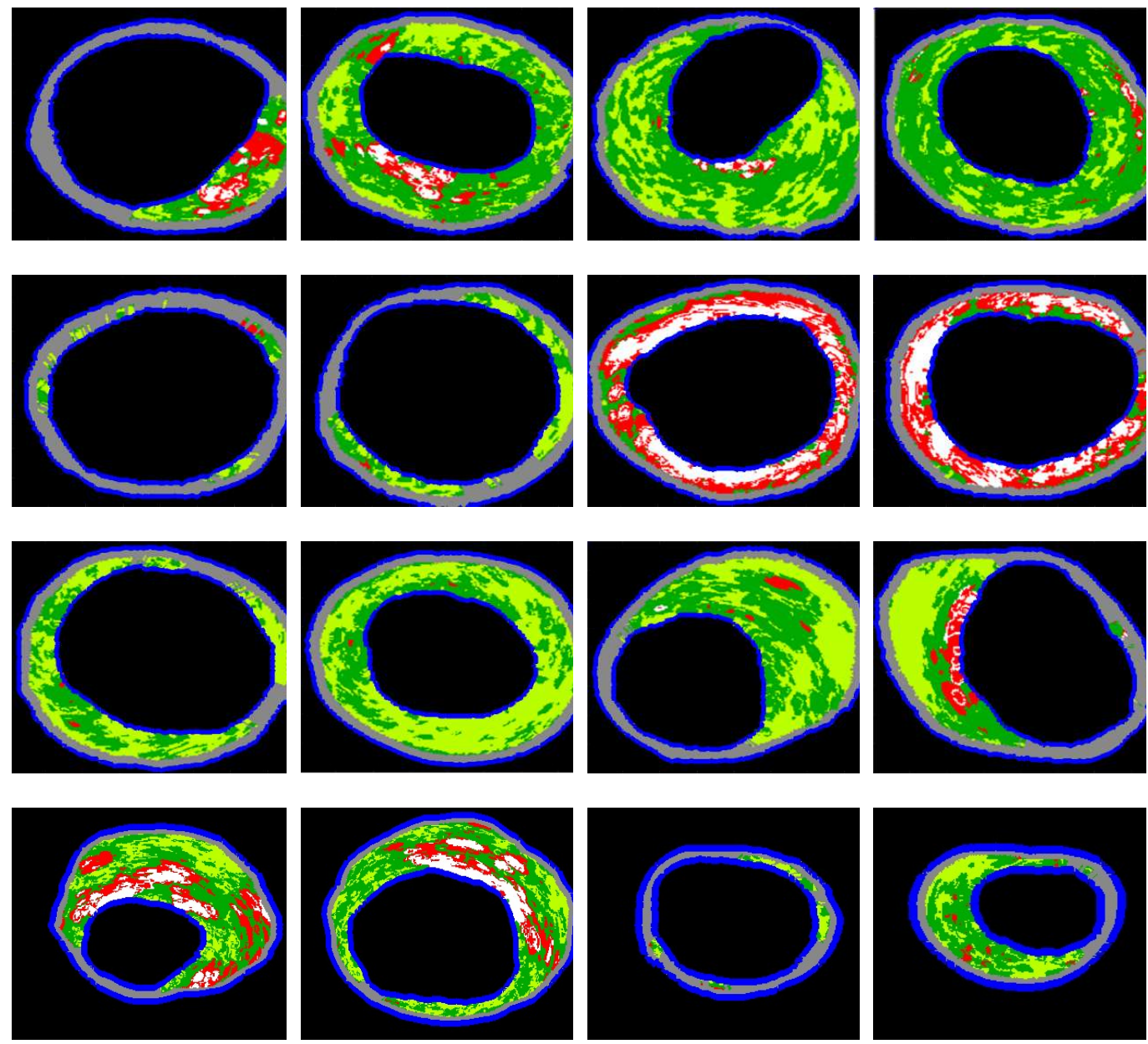

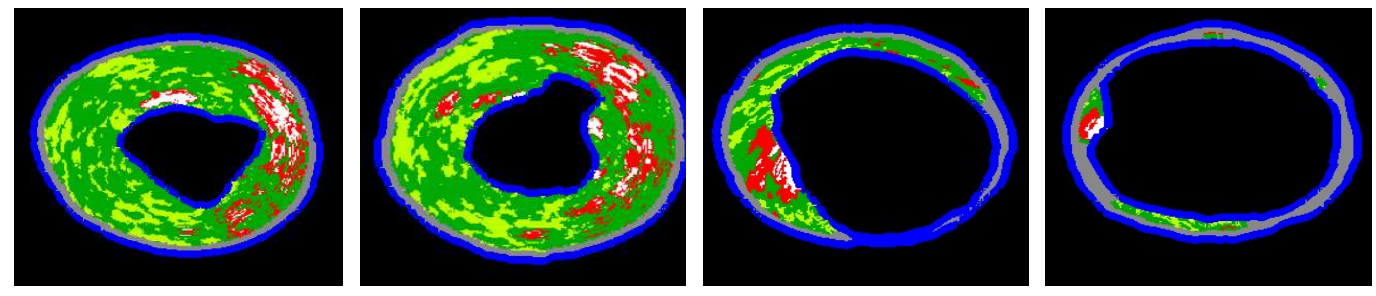

Figure 4 Plaque border detection by ACPB algorithm.

\subsection{Plaque Burden Measurement (PBM)}

In order to measure the plaque burden, coordinates of plaque border using ACPB algorithm are plotted in a new binary image to represent the plaque area $(P A)$. By applying the morphological operator, closed counter is then filled by ' 1 ' to show the vessel area $(V A)$. Lumen area $(L A)$ is obtained by means of subtracting the $V A$ from $P A$. The area of $V A$ and $L A$ are measured and stored in $f_{V A}$ and $f_{L A}$, respectively. Figure 5 illustrates the result of $\mathrm{PBM}$ algorithm. The Plaque burden $\left(f_{P B}\right)$ is computed based on the next formula [21]:

$$
f_{P B}=\frac{f_{V A}-f_{L A}}{f_{V A}} \times 100
$$

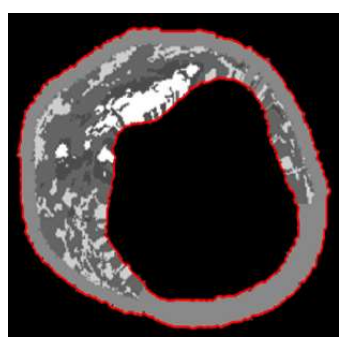

(a) Plaque border

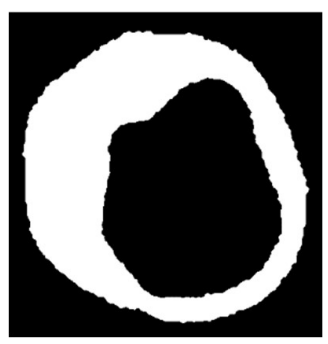

(b) Plaque area

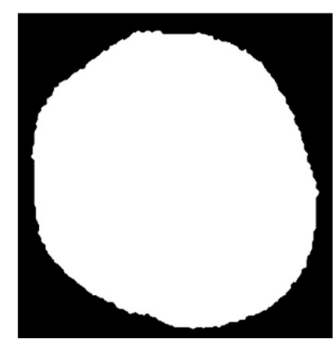

(c)Vessel area

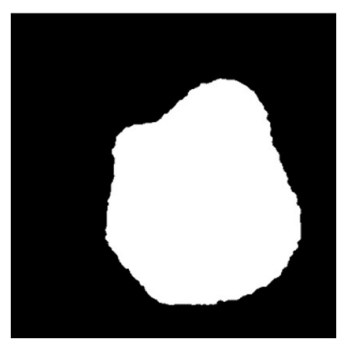

(a) Lumen area

Figure 5. Result of PBM algorithm.

\subsection{KMPSO-PMED Model for VH-IVUS Image Segmentation}

In our previous work, KMPSO-mED model was proposed to enhance the VH-IVUS image segmentation [17]. In this study, a new model namely Plaque based Minimum Euclidian Distance Plaque (PMED) is proposed to classify the pixels of testing image. This model executes the K-means algorithm on VH-IVUS image to generate the initial cluster centre and seed one initial particle of swarm. The rest initial particles are set randomly. The PSO is employed to generate the best solution for cluster centres. Based on the RBG colour of each cluster centre, generated clusters are labelled as red, white, dark green, and light green to represent the NC, DC, FI, and FF, respectively [16]. The outliers are detected and removed in NC and DC images [22]. The RGB values of DC, NC, FF, and FI images are extracted and saved into the color feature matrix namely $\mathrm{CF}$. The matching labels of plaque components, including $\mathrm{NC}=1, \mathrm{DC}=2, \mathrm{FF}=3$, and $\mathrm{FI}=4$ are determined and retained to the $L$ [16]. In order to classify each pixel of test image by PMED algorithm, CF is categorized into NC, DC, FF, and FI classes. In order to extract the PA of testing image, ACPB algorithm is applied. To perform the pixel wise classification, minimum Euclidian distance of pixel $x_{i}$ and four different classes are computed. The lowest minimum distance is provided the class label of $x_{i}$. Four plaque components are generated using the assigned label. The number of non-zero pixels of NC, DC, FF, and FI images are calculated to show the area features consisting of $f_{N C}, f_{D C}, f_{N C}, f_{F F}$, and $f_{F I}$, respectively. 


\subsection{Extracting Confluent Components (ECC) Algorithm}

To identify the confluent components as a mass with diameter of greater than 14 pixels in a 400×400 VH-IVUS image, NC image is binarized by the thresholding method [23]. In order to detect the NC segments, connected component labeling algorithm is then checked the 8neighborhood of withe pixels. If the segment's diameter $\geq 14$ pixels, then this segment is saved to the confluent NC (CNC) segments, else it is preserved in the scattered NC (SNC) segments [24]. Similarity, confluent DC (CDC) and micro calcification (MicroCa) are provided from the DC segments. The area of $\mathrm{CNC}, \mathrm{CDC}, \mathrm{SNC}$, and MicroCa segments are hold into the $f_{C N C}, f_{C D C}, f_{S N C}$, and $f_{\text {Microca, }}$ respectively. For extracting the confluent NC in contact with the lumen (CNCCL), share pixels between CNC and LB are determined. The entire process of Extracting Confluent Components (ECC) algorithm is enumerated as follows [16]:

Step 1: The NC image is binarized and then a binary image $B_{N C}$ is produced. Connected component labeling is performed for binary image $B_{N C}$ to produce a labeled matrix $L_{N C}$ of size $M \times N$ [25]. The $L_{N C}$ matrix involves the labels in range of 1 to $n$ representing each component. In order to divide the $n$ connected components, the coordinates $(\mathrm{x}, \mathrm{y})$ of each region of $L_{N C}$ are determined and their corresponding coordinates in $B_{N C}$ are then selected to generate a new binary image namely $S$ for each component.

Step 2: Therefore, binary images $S_{1}, S_{2}, \ldots S_{n}$ involving only one segment are generated [24]. Steps 3 to 6 are repeated $n$ times to calculate the diameter of each object and determine the CNC and SNC sets.

Step 3: The edge of segment $t_{i}$ belonging to the $S_{i}$ is extracted by edge detection algorithm. Let $X$ and $Y$ represent all $\mathrm{x}$ and $\mathrm{y}$ coordinates of the detected edge. The horizontal $h_{i}$ and vertical $v_{i}$ dimensions of the $X$ and $Y$ are provided by equations (6) and (7).

$h_{i}=\max (X)-\min (X)$

$v_{i}=\max (Y)-\min (Y)$

Sep 4: The diameter $D_{i}$ of segment $i$ is assumed to be the maximum value of $h_{i}$ and $v_{i}$ defined by:

$$
D_{\mathrm{i}}=\max \left(h_{i}, v_{i}\right)
$$

Step 5: If $D_{i} \geq 14$ pixels then segment $t_{i}$ is considered as CNC [26] and image $S_{i}$ renamed to $C N C_{i}$, otherwise it is called as $S N C_{i}$.

Step 6: The coordinates of segment $t_{i}$ in $C N C_{i}$ are stored in a 2D matrix $C_{i}$. The Coordinates of LB is then searched to find the possible shared points with $C_{i}$. If there is a shared point between LB and $C_{i}$, then it can be concluded that $C_{i}$ is in contact with the lumen and add to CNCCL segments.

Step 7: When the iteration is completed, the area of CNC images and SNC images are calculated by the following equation. Where, $b(i, j)$ with $\mathrm{M}$ rows and $\mathrm{N}$ columns consisting of ' 1 ' for white pixels and ' 0 ' for black pixels.

$$
\text { Area }=\sum_{i=1}^{M} \sum_{j=1}^{N} b(i, j)
$$

Step 8: The sum of all areas obtained by CNC and SNC images are stored into fCNC and $f_{S N C}$, respectively. Moreover, the number of CNCCL segments are stored in $f_{C N C C L}$. Figure 6 depicts the binary segments of CNCCL for a VH-IVUS image. The cyan pixels represent the lumen border, and white arrows illustrate the share points between lumen border and CNC. 


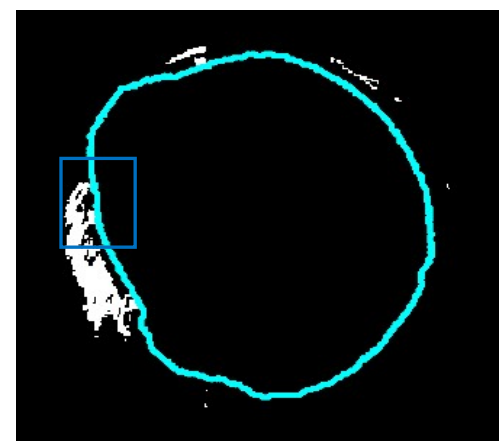

(a)

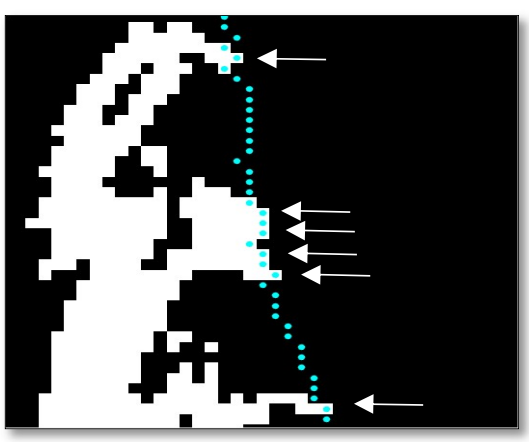

(b)

Figure 6 The CNCCL segments, (a) original image, (b) zoomed image.

The results of applying proposed algorithms for extracting confluent component are shown in Figure 7. This figure involves five columns to represent the VH-IVUS, NC, CNC, CNCCL, SNC, DC, CDC, and MicroCa images.

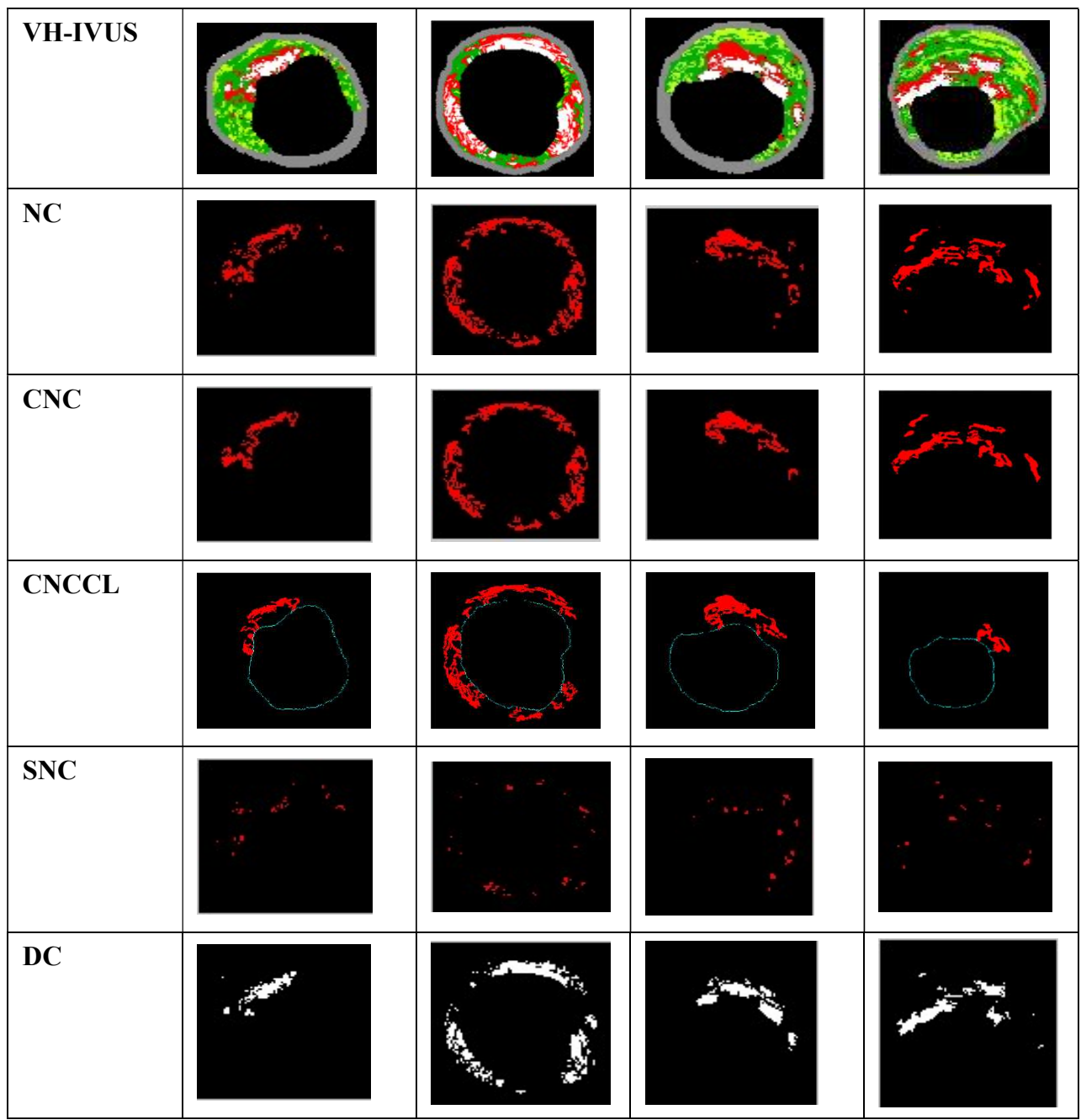




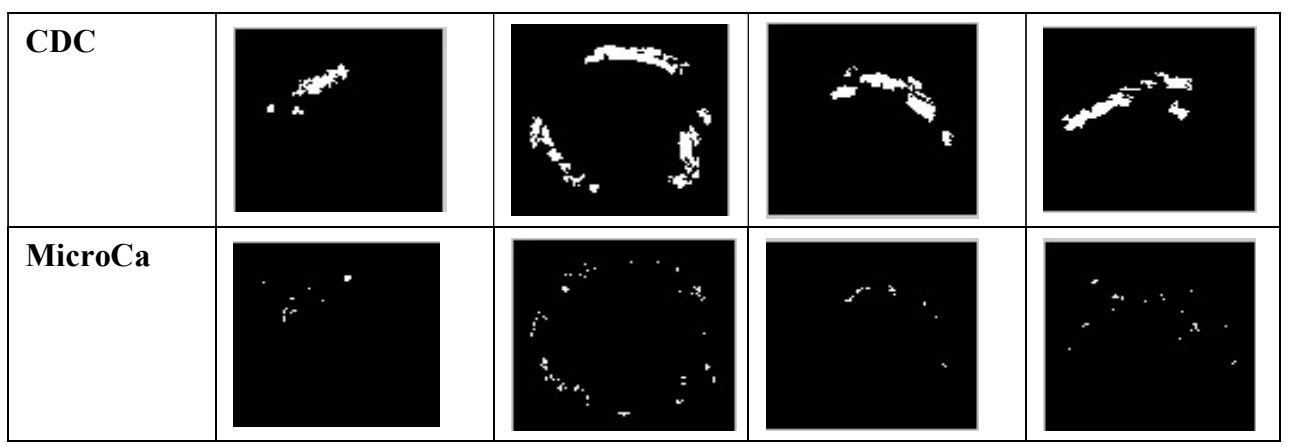

Figure 7 Result of ECC algorithms in VH-IVUS images (Note: NC: Necrotic core, CNC: Confluent NC, CNCCL: CNC in contact with the lumen, SNC: Scattered Necrotic Core, DC: Dense calcium, CDC: Confluent DC, and MicroCa: Micro Calcification).

\subsection{Determining NC Layering (DNCL) Algorithm}

TCFA is categorized according to the presence of CNC layers [23]. The DNCL algorithm is developed to identify the number of NC layers. Firstly, radial lines are drawn from the center of image in anti-clockwise direction to determine the pixels that are share between drawn lines and $\mathrm{CNC}$ segments. If one of the drawn line has share pixels with one segment, it means that NC involve only one layer. At the end, the total of NC layers is calculated and represented as $f_{N C L}$. Similarity, DC layers are determined and stored in $f_{D C L}$. The steps 1 to 4 are repeated $m$ times to calculate the NC layers [16].

Step 1: The images $C N C_{1}, C N C_{2}, \ldots . . C N C_{m}$ are put together in one binary image as $C N C$. The $x$ and $y$ coordinates of segment $t_{i}$ in $C N C_{i}$ is stored in a $2 \mathrm{D}$ matrix namely $C_{i}$ (m: number of CNC segments).

Step 2: The $n$ radials lines $R L=\left\{r l, \ldots . r l_{n}\right\}$ which start from the center of CNC image are drawn using. By employing the Bresenham's line algorithm, all pixels belonging to the each line are represented as $r l_{i}=\left\{p_{1} \ldots p_{n}\right\}$ [27].

Step 3: The points belonging to the line $r l_{i}$ are then searched to find the possible shared points with $C_{j}$. If there is a shared point, it means that this line passing through $C_{j}$ that is correspond to the segmentj. $P S_{i}$.

Step 4: The number of segments that $r l_{i}$ passes through them are retrieved and stored into the

Step 5: In order to identify the number of NC layers, the maximum value of $P S_{1}, P S_{2}, \ldots, P S_{n}$ is computed and stored as $f_{N C L}$ feature.

Drawing $n$ radial lines over the CNC image is presented in Figure 8. The red lines represent the radial lines and the white areas depicts the confluent NC segments in binary image. The zoom area indicated by the blue box in Figure 7 (a). As illustrated in this figure, radial lines pass through two segments. Therefore, the confluent NC has multiple layers that represent the previous rupture. Figure 9 presents the $\mathrm{x}$ and $\mathrm{y}$ coordinates (red color cells) of the shared points belonging to the radial line and coordinates of the CNC segment (white cells). 


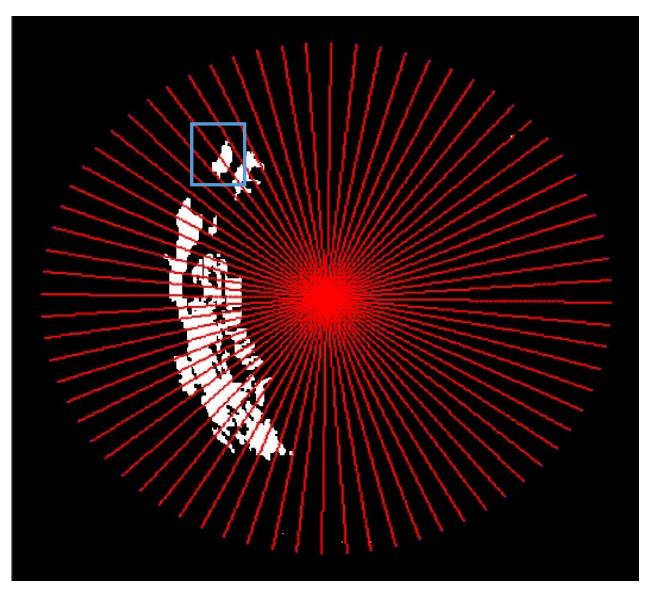

(a)

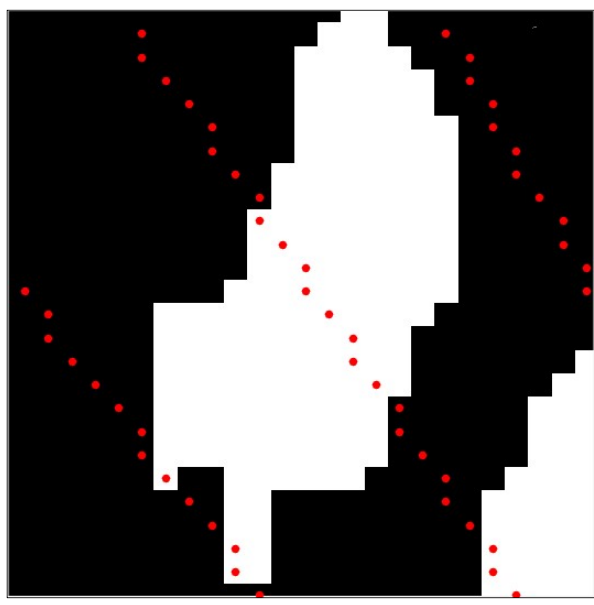

(b)

Figure 8 (a) Drawn lines from the center of image and (b) zoomed area, red pixels illustrate the drawn radial lines and the white areas depict the CNC segments in binary image.

$\begin{array}{llllll}100,132 & 100,133 & 100,134 & 100,135 & 100,136 & 100,137 \\ 101,132 & 101,133 & 101,134 & 101,135 & 101,136 & 101,137 \\ 102,132 & 102,133 & 102,134 & 102,135 & 102,136 & 102,137 \\ 103,132 & 103,133 & 103,134 & 103,135 & 103,136 & 103,137 \\ 104,132 & 104,133 & 104,134 & 104,135 & 104,136 & 104,137 \\ 105,132 & 105,133 & 105,134 & 105,135 & 105,136 & 105,137 \\ 106,132 & 106,133 & 106,134 & 106,135 & 106,136 & 106,137 \\ 107,132 & 107,133 & 107,134 & 107,135 & 107,136 & 107,137\end{array}$

Figure 9 The $\mathrm{x}$ and y coordinates of shared points between radial lines and segment of Figure 8 .

\subsection{Statistical Analysis}

Extracted features construct the VH-IVUS features as FV-VH=\{fNC, $f_{D C}, f_{F I}, f_{F F}, f_{N C C L}, f_{C N C}, f_{C N C}$, $\left.f_{C N C C L}, f_{C D C}, f_{N C L}, f_{D C L}, f_{M i c r o C a}, f_{S N C}, f_{P A}, f_{V A}, f_{L A,} f_{P B}\right\}$. In order to perform the t-test analysis, geometric features are categorized into three classes: PIT, TCFA, and CaTCFA based on the plaque type classification criteria. Mean, standard deviation (STD), and p-value of all 17 geometrical features are then calculated [28]. Table 1 depicts the t-test results (mean \pm STD) for three plaque types consisting of PIT, TCFA, and CaTCFA. Regarding the results, p-value $<0.0001$ are obtained for all features that indicate the discriminating capability of geometric features [29]. 
Table 1 T-test results (Mean \pm STD and p-value).

\begin{tabular}{ccccc}
\hline \multirow{2}{*}{ FV-VH } & \multicolumn{3}{c}{ Mean \pm STD } & \multirow{2}{*}{ p-value } \\
\cline { 2 - 4 } & PIT & TCFA & CaTCFA & \\
\hline$f_{N C}$ & $1.12 \pm 1.27$ & $6.18 \pm 2.45$ & $13.34 \pm 3.40$ & $<0.0001$ \\
\hline$f_{D C}$ & $0.35 \pm 0.71$ & $2.30 \pm 1.38$ & $7.34 \pm 1.61$ & $<0.0001$ \\
\hline$f_{F I}$ & $13.25 \pm 11.71$ & $16.82 \pm 11.62$ & $9.40 \pm 8.74$ & $<0.0001$ \\
\hline$f_{\mathrm{FF}}$ & $12.52 \pm 11.00$ & $10.87 \pm 8.71$ & $4.26 \pm 6.68$ & $<0.0001$ \\
\hline$f_{N C C L}$ & $0.02 \pm 0.02$ & $0.08 \pm 0.06$ & $0.21 \pm 0.09$ & $<0.0001$ \\
\hline$f_{D C C L}$ & $0.01 \pm 0.01$ & $0.04 \pm 0.03$ & $0.20 \pm 0.10$ & $<0.0001$ \\
\hline$f_{C N C}$ & $0.77 \pm 1.04$ & $5.60 \pm 2.34$ & $12.70 \pm 3.43$ & $<0.0001$ \\
\hline$f_{C N C C L}$ & $0.27 \pm 0.62$ & $3.72 \pm 2.69$ & $11.36 \pm 4.83$ & $<0.0001$ \\
\hline$f_{C D C}$ & $0.22 \pm 0.63$ & $1.75 \pm 1.45$ & $6.50 \pm 1.56$ & $<0.0001$ \\
\hline$f_{M i c r o C a}$ & $0.09 \pm 0.15$ & $0.53 \pm 0.20$ & $0.84 \pm 0.29$ & $<0.0001$ \\
\hline$f_{S N C}$ & $0.35 \pm 0.33$ & $0.57 \pm 0.28$ & $0.64 \pm 0.20$ & $<0.0001$ \\
\hline$f_{N C L}$ & $0.54 \pm 0.50$ & $1.00 \pm 0.00$ & $1.00 \pm 0.00$ & $<0.0001$ \\
\hline$f_{D C L}$ & $0.22 \pm 0.42$ & $0.95 \pm 0.23$ & $1.00 \pm 0.00$ & $<0.0001$ \\
\hline$f_{P A}$ & $28.29 \pm 16.36$ & $35.37 \pm 11.41$ & $41.55 \pm 4.03$ & $<0.0001$ \\
\hline$f_{V A}$ & $49.73 \pm 27.50$ & $64.53 \pm 21.32$ & $80.23 \pm 3.58$ & $<0.0001$ \\
\hline$f_{L A}$ & $21.44 \pm 15.14$ & $29.16 \pm 13.55$ & $38.68 \pm 4.85$ & $<0.0001$ \\
\hline$f_{P B}$ & $58.75 \pm 14.71$ & $56.35 \pm 10.73$ & $51.84 \pm 5.31$ & $<0.0001$ \\
\hline
\end{tabular}

In order to investigate the association between the geometric features and histopathological criteria, further analysis is performed for PIT, TCFA, and CaTCFA lesions using the mean values of the FV-VH features. The criteria of PIT, TCFA, and CaTCFA are summarized in Table 2.

Table 2 Criteria of PIT, TCFA, and CaTCFA lesion.

\begin{tabular}{ccccccc}
\hline Type & FF (\%) & FI (\%) & CNC (\%) & CDC (\%) & NCCL (\%) & PB (\%) \\
\hline PIT & $\geq 15$ & high & $<10$ & $<10$ & - & $\geq 40$ \\
\hline TCFA & - & - & $>10$ & $<10$ & $>0$ & $\geq 40$ \\
\hline CATCFA & - & - & $>10$ & $>10$ & $>0$ & $\geq 40$ \\
\hline
\end{tabular}

The mean value of the primary features consisting of $f_{F I}, f_{F F}, f_{C N C}$, and $f_{C D C}$ for PIT, TCFA, and CaTCFA classes are illustrated in Figure 10. The $x$-axis and y-axis indicate the features mean area $\left(\mathrm{mm}^{2}\right)$ of each feature, respectively.

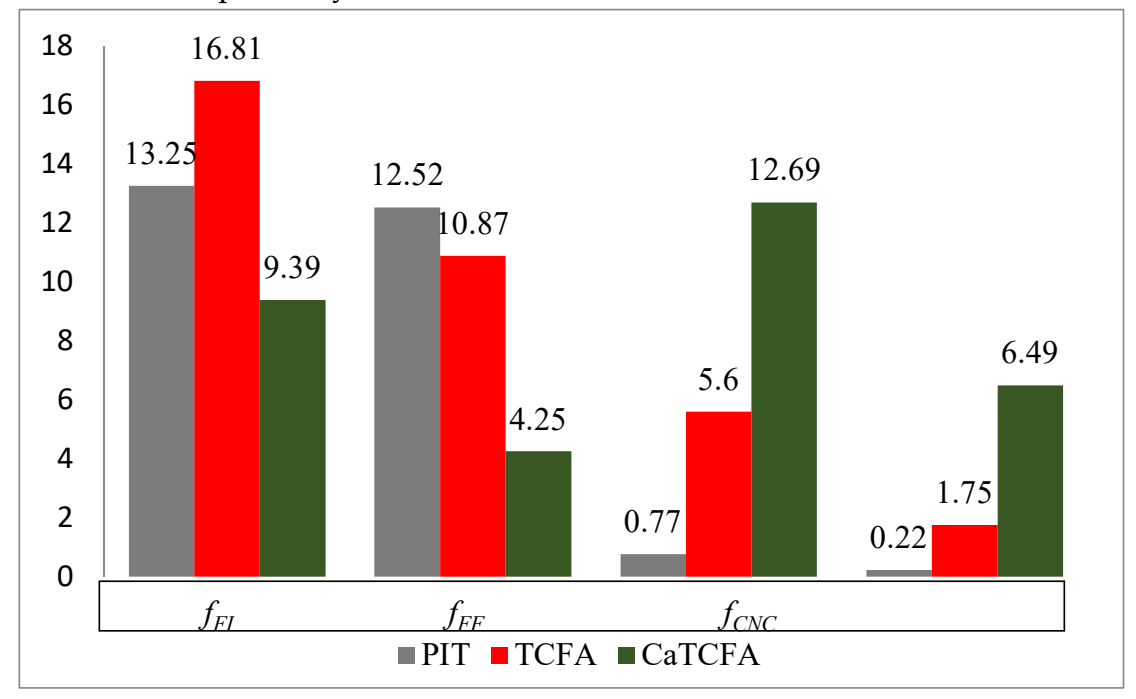

Figure 10 The mean of $f_{F I}, f_{F F}, f_{C N C}$, and $f_{C D C}$ features for PIT, TCFA, and CaTCFA classes.

The value of FF should be maximum for PIT class based on the Table 2. As shown in Figure 10, PIT achieves the maximum value of $f_{F F}$. Also, as depicted in Table 2 , for CaTCFA plaque, CDC $>10 \%$ and for others, CDC $<10 \%$, so the CDC value of CaTCFA must be greater than the TCFA and CaTCFA. As depicted in this figure, $f_{C D C}$ of $C a T C F A$ has the highest value among the other classes. As illustrated 
in Table 2 for TCFA and CaTCFA lesions, CNC $>10 \%$, but for PIT CNC $<10 \%$, so the minimum value of $\mathrm{CNC}$ should be provided for PIT lesion. Figure 10 demonstrates that $f_{C N C}$ of PIT obtains the lowest value. Therefore, the requirements are satisfied by the mean values of $f_{F I}, f_{F F}, f_{C N C}$, and $f_{C D C}$ features.

Figure 11 illustrates the mean $\left(\mathrm{mm}^{2}\right)$ of $f_{F I}, f_{F F}, f_{C N C}$, and $f_{C D C}$, and $f_{P A}$ in for PIT lesions. As described in Table 2, PIT involve the mixture of FI and FF ( FF $\geq 15 \%$ ). The results presented in this figure fulfills these criteria as the mean of $f_{F I}$ and $f_{F F}$ are superior to $f_{C N C}$ and $f_{C D C}$. Moreover, based on Table 2, the mean of CNC and CDC should less than $10 \%$ of the plaque area that is satisfied by the $f_{C N C}<10 \% f_{P A}$ and $f_{C D C}<10 \% f_{P A}$ in this figure.

Figure 12 depicts the mean $\left(\mathrm{mm}^{2}\right)$ of $f_{F I}, f_{F F}, f_{C N C}$, and $f_{C D C}, f_{N C C L}, f_{P B}$ and $f_{P A}$ features for TCFA lesions. According to the Table 2, three features are important for TCFA detection, including CNC $>10 \%, \mathrm{NCCL}>0$, and CDC $<10 \%$. As illustrated in the Figure 12 , the mean values of $f_{C N C}>10 \% f_{P A}, f_{N C C L}>$ 0 , and $f_{P B}>40 \%$. Therefore, given criteria of TCFA detection are fulfilled.

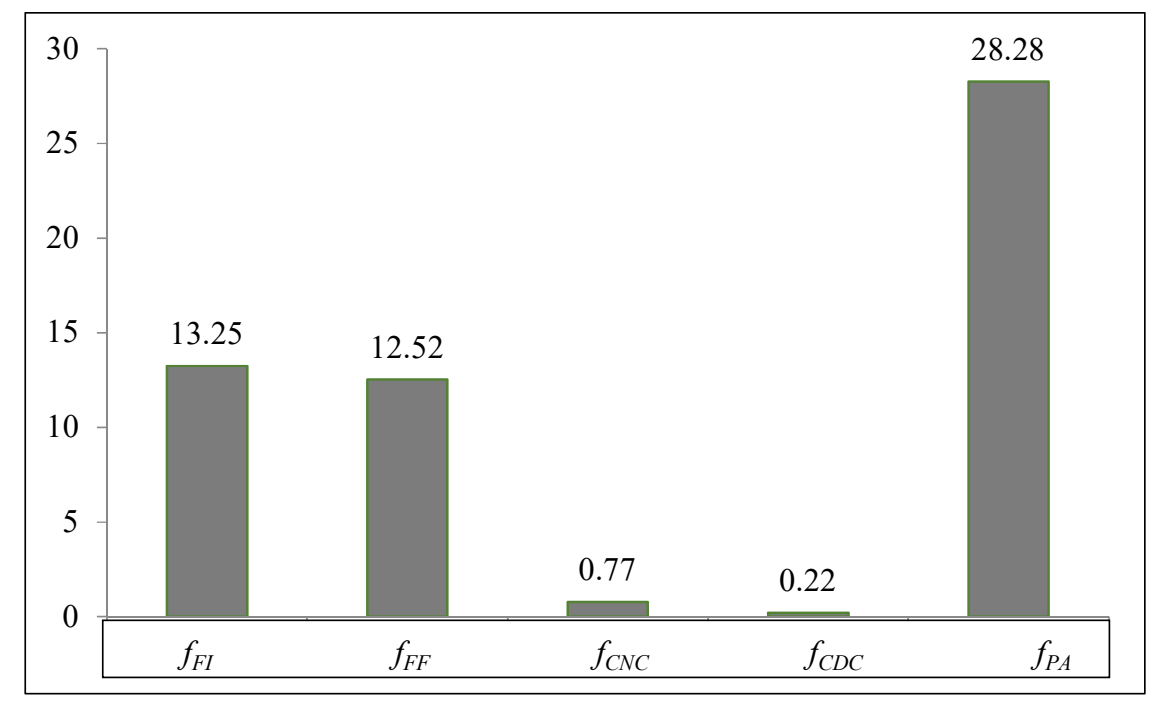

Figure 11 The mean $\left(\mathrm{mm}^{2}\right)$ of $f_{F I}, f_{F F}, f_{C N C}$, and $f_{C D C}$, and $f_{P A}$ for PIT .

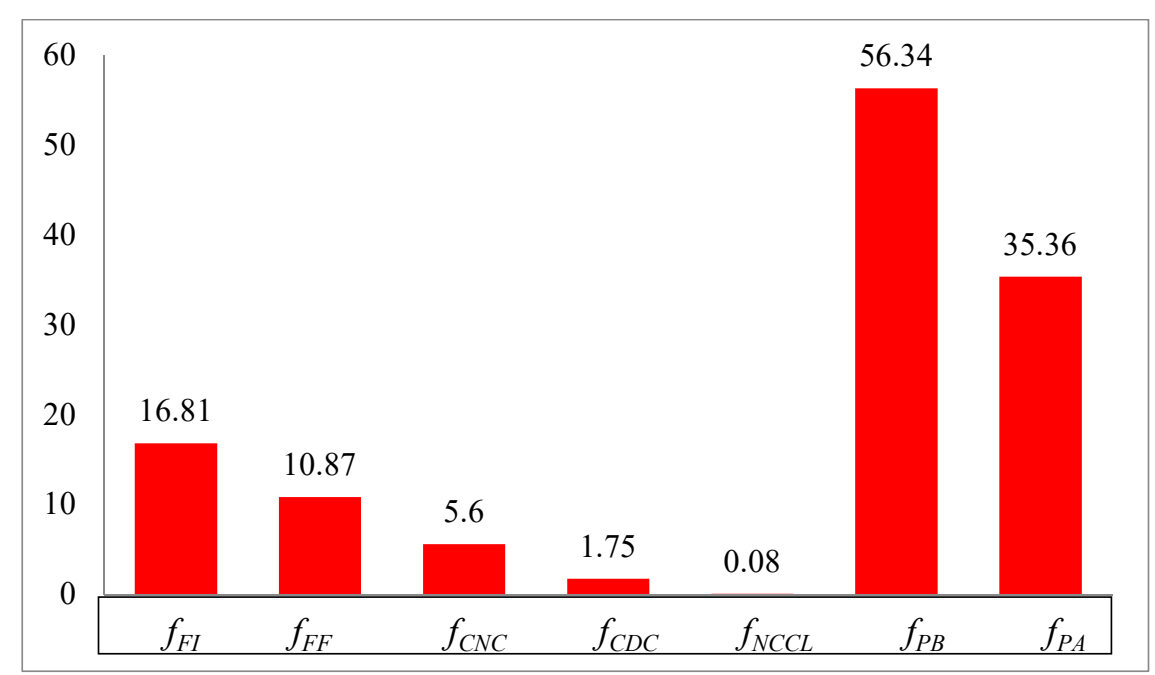

Figure 12 The mean $\left(\mathrm{mm}^{2}\right)$ of $f_{F I}, f_{F F}, f_{C N C}$, and $f_{C D C}, f_{N C C L}, f_{P B}$ and $f_{P A}$ for TCFA. 
Figure 13 illustrates mean of $\left(\mathrm{mm}^{2}\right) f_{F I}, f_{F F}, f_{C N C}$, and $f_{C D C}, f_{N C C L}, f_{B P}$ and $f_{P A}$ features for CaTCFA lesions. According to the Table 2, four conditions are presented for CaTCFA detection, including $\mathrm{CNC}>10 \%, \mathrm{CDC}>10 \%, \mathrm{NCCL}>0$ and $\mathrm{PB} \geq 40 \%$. As illustrated in this figure, $f_{C N C}>10 \% f_{P A}, f_{C D C}>10 \%$ $f_{P A}, f_{N C C L}>0$, and $f_{P B} \geq 40 \%$. So, the criteria of CaTCFA are satisfied.

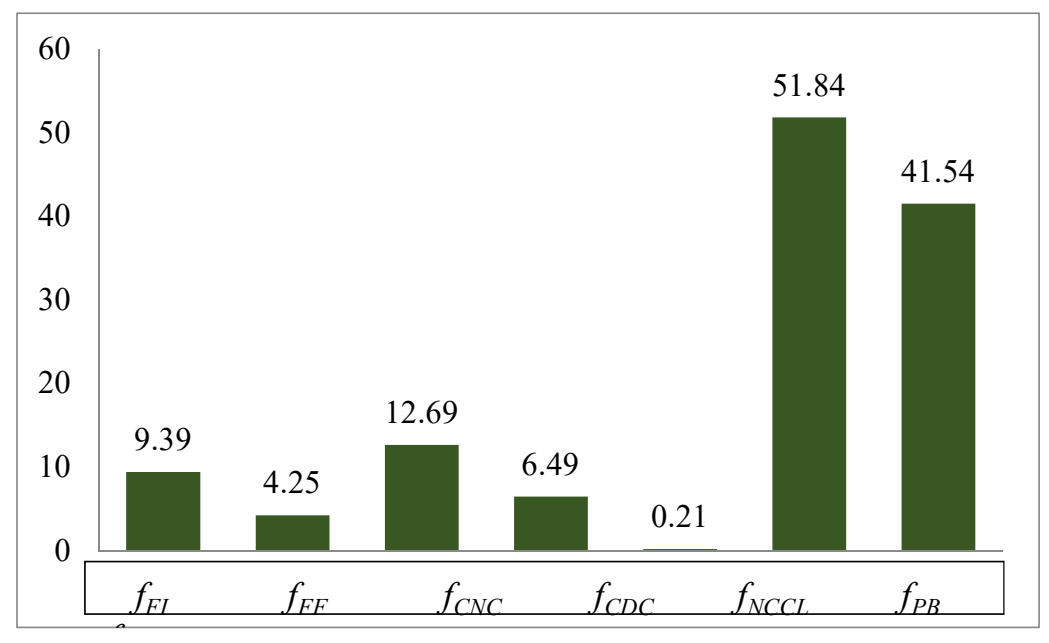

Figure 13 The mean of $f_{F I}, f_{F F}, f_{C N C}$, and $f_{C D C}, f_{N C C L}, f_{P B}$ and $f_{P A}$ features for CaTCFA.

The obtained results indicate that the geometric features that are categorized in PIT, TCFA, and CaTCFA considerably represent the histological criteria of plaque type classification.

\subsection{IVUS features}

Volcano's software can be provided 12 features of gold standard IVUS modality for plaque characterization [30]. The area of lumen, fibrous, fibro lipidic, lipid core, calcified, and Media are measured. Moreover, minimum, maximum, and average of lumen diameter and vessel diameter obtained. The area of external elastic membrane (EEM) or vessel is provided by the total area of plaque, lumen, and media [31,32]. Plaque burden is expressed as a percentage of the vessel area and computed by means of the difference between two parameters, including vessel area and lumen area [33].

\section{Results}

In this section, effects of the extracted features for classification of the plaque is explored.

\subsection{Data Set Labeling}

The data set of this study consisting of 566 Gray-scale IVUS and their corresponding VH-IVUS images were obtained from the 9 patients [35]. The feature extraction algorithms are implemented in MATLAB software and feature matrix namely FM of size 566×17are created from VH-IVUS images. Based on the existing criteria, FM matrix is labeled as " 1 " or " 0 " corresponding to the TCFA or non-TCFA plaque. Therefore, the size of FM is resized to $566 \times 18$ dimensions. TCFA is defined as a plaque with plaque burden $\geq 40 \%, \mathrm{CNC} \geq 10 \%, \mathrm{CNCCL} \geq 10 \%$, and $\mathrm{CDC}<10 \%[23,36]$.

The FM can be divided into different subsets to obtain the most significant and effective features of TCFA and achieve the desired accuracy. Therefore, histopathological features are considered to determine the different group of TCFA features. The feature set 1(FS1) is constructed using all FV$\mathrm{VH}$ features to represent the main criteria of plaque type and feature set 2 (FS2), feature set 3 (FS3), feature set 4 (FS4), and feature set 5 (FS5) are constructed based on the different definition of the TCFA plaque. A few studies have considered the multiple NC layers as a critical feature of TCFA plaque [26, 37, 38]. However, others did not consider the NC layers [14, 39]. Thus, to investigate the effect of NC layering on classification results, FS2 is built by excluding the NC layering features 
consisting of $f_{N C L}, f_{D C L}$. Moreover, some researchers consider a plaque burden $\geq 40 \%$ to detect the TCFA lesion [14, 39]. Therefore, FS3 is constructed by eliminating the plaque burden features consisting of $f_{P A}, f_{V A}, f_{L A}$, and $f_{P B}$ to investigate the effect of these features. Furthermore, different studies only introduce the basic features and ignore the plaque burden and layering features. Therefore, FS4 consisting of $f_{C N C}, f_{C D C}, f_{F I}, f_{F F}$ and $f_{N C C L}$ is established by eliminating the plaque burden features and layering features. In addition, FS5 is constructed by the plaque area features consisting of $f_{N C}, f_{D C}, f_{F I}$, $f_{F F}$. Table 3 illustrated different feature sets consisting of FS1, FS2, FS3, FS4, and FS5.

Table 3 Feature set (FS1: feature set 1, FS2: feature set 2, FS3: feature set 3, FS4: feature set 4, and FS5: feature set 5).

\begin{tabular}{cc}
\hline Feature set & Features \\
\hline FS1 & $f_{N C}, f_{D C}, f_{F I}, f_{F F}, f_{N C C L}, f_{D C C L}, f_{C N C}, f_{C N C C L}, f_{C D C}, f_{N C L}, f_{D C L}, f_{M i c r o C a}, f_{S N C}, f_{P A}, f_{V A,}, f_{L A}, f_{P B}$ \\
\hline FS2 & $f_{N C}, f_{D C}, f_{F I}, f_{F F}, f_{N C C L}, f_{C N C}, f_{C N C C L}, f_{C D C}, f_{N C L}, f_{D C L}, f_{M i c r o C a}, f_{S N C,}, f_{P A}, f_{V A}, f_{L A}, f_{P B}$ \\
\hline FS3 & $f_{N C}, f_{D C}, f_{F I}, f_{F F}, f_{N C C L}, f_{D C C L}, f_{C N C}, f_{C N C C L}, f_{C D C}, f_{N C L}, f_{D C L}, f_{M i c r o C a}, f_{S N C}$ \\
\hline FS4 & $f_{C N C}, f_{C D C}, f_{F I}, f_{F F}, f_{N C C L}$ \\
\hline FS5 & $f_{N C}, f_{D C}, f_{F I}, f_{F F}$ \\
\hline
\end{tabular}

\subsection{Experimental Setup}

The K-fold cross validation technique has been employed for evaluating the results of SVM classifier. The first k-1 parts are considered as the training set and the remaining one part is applied to evaluate the trained classifier [29]. Different kernels of SVM consisting of linear, quadratic, polynomial, rbf, and mlp are employed. To evaluate the performance, accuracy, sensitivity, and specificity are calculated [41]. The features are normalized by the zscore function due to the various range of feature values [43].

\subsection{TCFA Classification by VH-IVUS Images}

Regarding the experimental setup, $70 \%$ and $30 \%$ of FM matrix of size $566 \times 18$ are considered as training and testing sets, respectively. Therefore, FMTrain of size $397 \times 18$ and FMTest of size $169 \times 18$ are generated. The confusion matrix is calculated, and the averages accuracy, sensitivity, and specificity parameters derived from 10 runs are calculated. Performance are measured using different groups of feature sets. The result of FS1, FS2, FS3, and FS4 are illustrated in Table 4.

Table 4. The result of different SVM kernels using VH-IVUS features.

\begin{tabular}{|c|c|c|c|c|c|c|}
\hline Feature Set & Measurement & Linear & Quadratic & Polynomial & Rbf & Mlp \\
\hline \multirow{3}{*}{ FS1 } & Accuracy & 97.63 & 98.82 & 98.22 & 95.86 & 91.12 \\
\hline & Sensitivity & 100 & 100 & 100 & 100 & 100 \\
\hline & Specificity & 97.01 & 98.51 & 97.76 & 94.78 & 88.81 \\
\hline \multirow{3}{*}{ FS2 } & Accuracy & 98.22 & 98.22 & 98.42 & 95.46 & 89.94 \\
\hline & Sensitivity & 100 & 97.14 & 98.09 & 99.04 & 97.14 \\
\hline & Specificity & 97.76 & 98.50 & 98.50 & 94.52 & 88.05 \\
\hline \multirow{3}{*}{ FS3 } & Accuracy & 98.82 & 95.85 & 97.43 & 97.04 & 95.85 \\
\hline & Sensitivity & 100 & 88.57 & 94.28 & 99.04 & 97.14 \\
\hline & Specificity & 98.50 & 97.76 & 98.25 & 96.51 & 95.52 \\
\hline \multirow{3}{*}{ FS4 } & Accuracy & 97.6 & 97.04 & 97.23 & 95.06 & 90.53 \\
\hline & Sensitivity & 100 & 97.14 & 97.14 & 100 & 100 \\
\hline & Specificity & 97.01 & 97.01 & 97.26 & 93.78 & 88.05 \\
\hline \multirow{3}{*}{ FS5 } & Accuracy & 98.22 & 98.22 & 98.02 & 96.25 & 93.49 \\
\hline & Sensitivity & 100 & 100 & 99.04 & 97.14 & 97.14 \\
\hline & Specificity & 97.76 & 97.76 & 97.76 & 96.01 & 92.53 \\
\hline
\end{tabular}


Based on Table 4, the best result of accuracy is obtained by applying the quadratic kernel for FS1 and linear kernel for FS3. While the worst result is provided by FS2 using the mlp kernel. The highest sensitivity is achieved for FS1 and the lowest result is provided by FS3 using the quadratic kernel. In addition, FS1 illustrates the best result of specificity by employing the quadratic kernel, whereas the worst result is achieved by FS2 and FS4 using the linear kernel.

\subsection{TCFA Classification by fusion of VH-IVUS and IVUS Images}

In order to investigate the power of various feature sets, IVUS and VH-IVUS features are fused. Consider FV-VH and IVUS features are represented as FV-VH $=\left\{v h_{1}, v h_{2}, \ldots v h_{17}, l\right\}$ and FV-IVUS $=\{$ ivus1,ivus2,...ivus12 $\}$, respectively, where $l$ represents the label of vector. Table 5 depicts the results of combination of IVUS features and FS1, FS2, FS3, FS4, and FS5 in terms of accuracy, sensitivity, and specificity.

Table 5. The result of different SVM kernels using VH-IVUS and IVUS features.

\begin{tabular}{|c|c|c|c|c|c|c|}
\hline $\begin{array}{c}\text { Feature } \\
\text { Set }\end{array}$ & Measurement & Linear & Quadratic & Polynomial & Rbf & Mlp \\
\hline \multirow{3}{*}{ IVUS } & Accuracy & 88.75 & 69.23 & 83.23 & 88.75 & 22.48 \\
\hline & Sensitivity & 100 & 54.28 & 72.38 & 47.61 & 100 \\
\hline & Specificity & 85.82 & 73.13 & 86.06 & 99.50 & 2.23 \\
\hline \multirow{3}{*}{ FS1-IVUS } & Accuracy & 96.44 & 94.08 & 95.85 & 88.36 & 67.45 \\
\hline & Sensitivity & 100 & 82.85 & 91.42 & 48.57 & 100 \\
\hline & Specificity & 95.52 & 97.01 & 97.01 & 98.75 & 58.95 \\
\hline \multirow{3}{*}{ FS2-IVUS } & Accuracy & 97.04 & 96.44 & 96.44 & 91.51 & 37.86 \\
\hline & Sensitivity & 100 & 85.71 & 89.52 & 66.66 & 100 \\
\hline & Specificity & 96.26 & 99.25 & 98.25 & 98.01 & 21.64 \\
\hline \multirow{3}{*}{ FS3-IVUS } & Accuracy & 98.22 & 95.85 & 96.64 & 90.92 & 73.37 \\
\hline & Sensitivity & 100 & 91.42 & 93.33 & 62.85 & 100 \\
\hline & Specificity & 97.76 & 97.01 & 97.51 & 98.25 & 66.41 \\
\hline \multirow{3}{*}{ FS4-IVUS } & Accuracy & 98.22 & 98.22 & 94.47 & 90.13 & 30.76 \\
\hline & Sensitivity & 100 & 97.14 & 81.90 & 60 & 100 \\
\hline & Specificity & 97.76 & 98.50 & 97.76 & 98.01 & 12.68 \\
\hline \multirow{3}{*}{ FS5-IVUS } & Accuracy & 99.40 & 98.22 & 93.68 & 88.56 & 30.76 \\
\hline & Sensitivity & 100 & 97.14 & 76.19 & 49.52 & 100 \\
\hline & Specificity & 99.25 & 98.50 & 98.25 & 98.75 & 12.68 \\
\hline
\end{tabular}

Based on Table 5, the best results of accuracy is obtained by fusion of IVUS and FS5 by applying the linear kernel, while the worst result is provided by IVUS features. In addition, the best result of sensitivity is provided by employing the linear kernel for all feature sets, while the worst result is achieved by IVUS features using rbf kernel. The highest and the lowest specificity are achieved using the IVUS features by rbf and mlp kernel, respectively. Figure 14 illustrates the maximum accuracy result of different feature sets. 


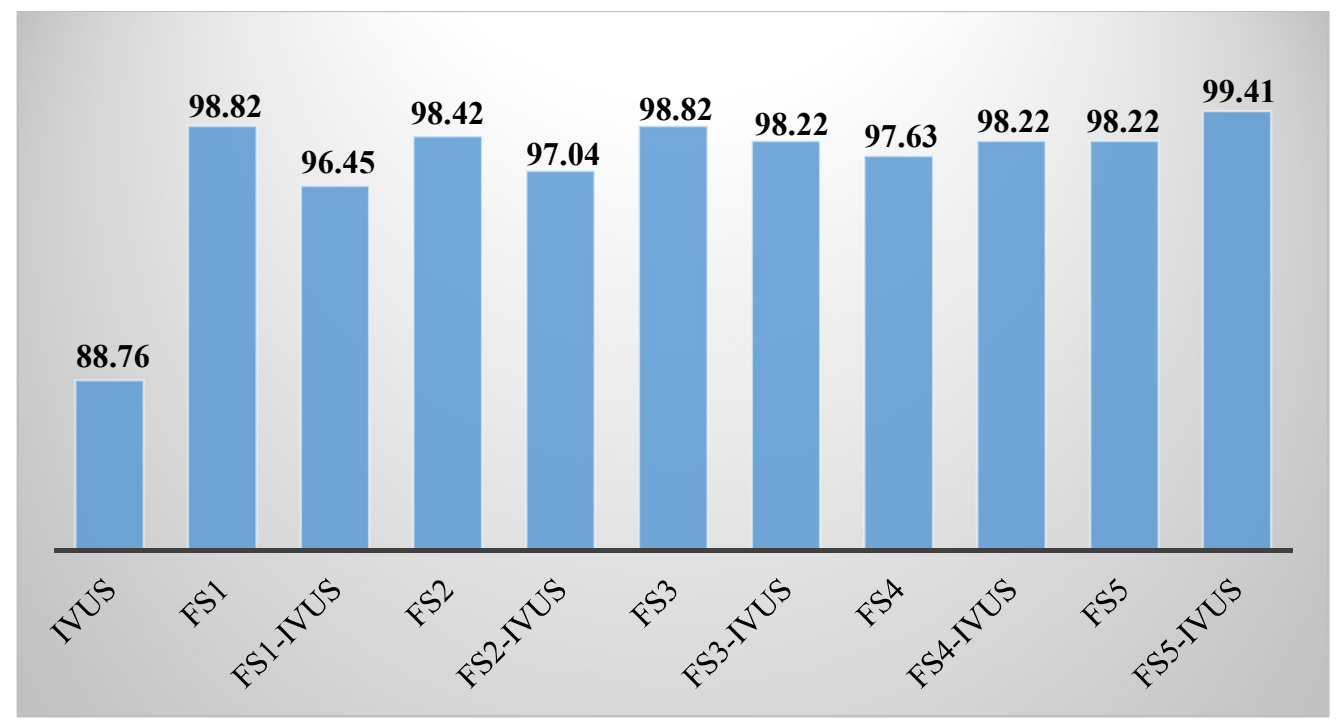

Figure 14 The maximum accuracy result of different feature sets.

As depicted in this figure, the highest and lowest results are achieved by IVUS and FS5-IVUS features, respectively. The fusion of FS1, FS2, and FS3 with IVUS features illustrate the lower result than FS1, FS2, and FS3 features, respectively. While, the fusion of FS4 and FS5 with IVUS show the improved results rather than FS4 and FS5.

\section{Discussion}

Automatic plaque type classification need to moderate the examiner observer fault [44]. However, because of different criteria for plaque classification, the definition of a TCFA plaque is not clearly standardized [14,37]. Moreover, there are different definitions for plaque type classification, but the effects of different plaque definitions have not been studied [14]. Therefore, the results of classification are different $[45,46]$. Regarding the clinical research, vessel morphology changes over the time due to the dynamic nature of coronary artery. Nevertheless, intravascular images cannot fully assess the morphology of the plaque. Therefore, imaging-derived predictors to determine the clinical outcomes and assess more reliably plaque morphology should be thoroughly fused.

\section{Conclusions}

In this study, automatic detection of TCFA plaque by means of the fusion of IVUS and VH-IVUS images is presented. A new algorithm is developed to extract the plaque borders based on the level set method. Based on the histopathological studies, extracted features were divided into different subsets to obtain the most significant and effective features of TCFA. Highest accuracy of SVM classifier is achieved using the integrated features of IVUS and VH-IVUS images rather than IVUS features. A prognostic assessment of vulnerable plaques contributes to the identification of high-risk patients. However, further research is required to investigate the concept of vulnerable plaque.

\section{References}

1. Zhang, L., et al. Location-specific prediction of vulnerable plaque using IVUS, virtual histology, and spatial context. in IEEE 13th International Symposium on Biomedical Imaging (ISBI). 2016. IEEE.

2. Dulohery, K., et al., Evaluation of coronary stenosis with the aid of quantitative image analysis in histological cross sections. Journal of forensic and legal medicine, 2012.

3. Katouzian, A., et al., A state-of-the-art review on segmentation algorithms in intravascular ultrasound (IVUS) images. IEEE Transactions on Information Technology in Biomedicine 2012. 16(5): p. 823-834.

4. Nair, A., et al., Assessing spectral algorithms to predict atherosclerotic plaque composition with normalized and raw intravascular ultrasound data. Ultrasound Med. Biol., 2001. 27(10): p. 1319-1331 
5. Papaioannou, T.G., et al., Quantification of new structural features of coronary plaques by computational post-hoc analysis of virtual histology-intravascular ultrasound images. Computer methods in biomechanics and biomedical engineering, 2014. 17(6): p. 643-651.

6. König, A., et al., Technology insight: in vivo coronary plaque classification by intravascular ultrasonography radiofrequency analysis. Nature Clinical Practice Cardiovascular Medicine, 2008. 5(4): p. 219-229.

7. Obaid, D.R., et al., Identification of coronary plaque sub-types using virtual histology intravascular ultrasound is affected by inter-observer variability and differences in plaque definitions. Circulation: Cardiovascular Imaging, 2012. 5(1): p. 86-93.

8. Cilla, M., Mechanical effects on the atheroma plaque appearance, growth and vulnerability. 2013.

9. Tang, D., et al., Local maximal stress hypothesis and computational plaque vulnerability index for atherosclerotic plaque assessment. Annals of biomedical engineering, 2005. 33(12): p. 1789-1801.

10. Margolis, M.P., et al., Automated lesion analysis based upon automatic plaque characterization according to a classification criterion, 2009, Google Patents.

11. Fernando J R Sales, J.L.A.A.F., Breno A A Falcão, Sérgio S Furuie, Pedro A Lemos. A computational tool for coronary atherosclerotic plaque analysis of Virtual Histology images. in Computing in Cardiology, 2010. 2010. IEEE.

12. Kubo, T., et al., The dynamic nature of coronary artery lesion morphology assessed by serial virtual histology intravascular ultrasound tissue characterization. Journal of the American College of Cardiology, 2010. 55(15): p. 1590-1597.

13. Czopek, K., J. Legutko, and J. Jąkała. Quantitative assessment for confluent plaque area related to diagnostic IVUS/VH images. in Computing in Cardiology, 2011. 2011. IEEE.

14. Obaid, D.R., et al., Identification of coronary plaque sub-types using virtual histology intravascular ultrasound is affected by inter-observer variability and differences in plaque definitions. Circ Cardiovasc Imaging, 2012. 5(1): $\mathrm{p}$. 86-93.

15. Zhang, L., et al., Prospective prediction of Thin-Cap Fibroatheromas from baseline Virtual Histology Intravascular Ultrasound data, in Medical Image Computing and Computer-Assisted Intervention-MICCAI 2015. 2015, Springer. p. 603-610.

16. Rezaei, Z., et al., Automatic Plaque Segmentation based on hybrid Fuzzy Clustering and $k$ Nearest Neighborhood using Virtual Histology Intravascular Ultrasound Images. Applied Soft Computing, 2016. 53.

17. Rezaei, Z., et al., Thin Cap Fibroatheroma Detection in Virtual Histology Images Using Geometric and Texture Features. Applied Sciences, 2018. 8(9): p. 1632.

18. Singh, M., S. Singh, and S. Gupta, An information fusion based method for liver classification using texture analysis of ultrasound images. Information Fusion, 2014. 19: p. 91-96.

19. Zhang, K., et al., Active contours with selective local or global segmentation: a new formulation and level set method. Image and Vision computing, 2010. 28(4): p. 668-676.

20. Rad, A.E., et al., Morphological region-based initial contour algorithm for level set methods in image segmentation. Multimedia Tools and Applications, 2017. 76(2): p. 2185-2201.

21. Papadopoulou, S.-L., et al., Assessment of atherosclerotic plaques at coronary bifurcations with multidetector computed tomography angiography and intravascular ultrasound-virtual histology. European Heart JournalCardiovascular Imaging, 2012: p. jes083.

22. Mira, A., D. Bhattacharyya, and S. Saharia, RODHA: robust outlier detection using hybrid approach. American Journal of Intelligent Systems, 2012. 2(5): p. 129-140.

23. Maehara, A., et al., Definitions and methodology for the grayscale and radiofrequency intravascular ultrasound and coronary angiographic analyses. JACC: Cardiovascular Imaging, 2012. 5(3s1): p. S1-S9.

24. Sakata, K., et al., Intravascular ultrasound appearance of scattered necrotic core as an index for deterioration of coronary flow during intervention in acute coronary syndrome. Heart and vessels, 2012. 27(5): p. 443-452.

25. Jain, R., R. Kasturi, and B.G. Schunck, Machine vision. Vol. 5. 1995: McGraw-Hill New York.

26. Konig, A., et al., Intravascular ultrasound radiofrequency analysis of the lesion segment profile in ACS patients. Clin Res Cardiol, 2010. 99(2): p. 83-91.

27. Wright, W.E., Parallelization of Bresenham's line and circle algorithms. Computer Graphics and Applications, 1990. 10(5): p. 60-67.

28. Acharya, U.R., et al., An integrated index for detection of Sudden Cardiac Death using Discrete Wavelet Transform and nonlinear features. Knowledge-Based Systems, 2015. 83: p. 149-158.

29. Acharya, U.R., et al., Ultrasound-based tissue characterization and classification of fatty liver disease: A screening and diagnostic paradigm. Knowledge-Based Systems, 2015. 75: p. 66-77.

30. Kubo, T., et al., Virtual histology intravascular ultrasound compared with optical coherence tomography for identification of thin-cap fibroatheroma. International heart journal, 2011. 52(3): p. 175-179.

31. Nissen, S.E. and P. Yock, Intravascular Ultrasound Novel Pathophysiological Insights and Current Clinical Applications. Circulation, 2001. 103: p. 604-616. 
32. Ouldzein, H., et al., Plaque rupture and morphological characteristics of the culprit lesion in acute coronary syndromes without significant angiographic lesion: Analysis by intravascular ultrasound. Annales de Cardiologie et d'Angéiologie, 2012. 61: p. 20-26.

33. Vazquez-Figueroa, J.G., et al., Prospective Validation that Vulnerable Plaque Associated with Major Adverse Outcomes Have Larger Plaque Volume, Less Dense Calcium, and More Non-Calcified Plaque by Quantitative, ThreeDimensional Measurements Using Intravascular Ultrasound with Radiofrequency Backscatter Analysis. Journal of cardiovascular translational research, 2013. 6(5): p. 762-771.

34. Ain, Q., M.A. Jaffar, and T.-S. Choi, Fuzzy anisotropic diffusion based segmentation and texture based ensemble classification of brain tumor. Applied Soft Computing, 2014. 21: p. 330-340.

35. Taki, A., et al., An IVUS image-based approach for improvement of coronary plaque characterization. Computers in Biology and Medicine, 2013. 43: p. 268-280.

36. Kim, S.-J., et al., Reproducibility of in vivo measurements for fibrous cap thickness and lipid arc by oct. JACC: Cardiovascular Imaging, 2012. 5(10): p. 1072-1074.

37. Cascón-Pérez, J.D., et al., Characteristics of culprit atheromatous plaques obtained in vivo by intravascular ultrasound radiofrequency analysis: Results from the CULPLAC study. American Heart Journal, 2013.

38. Zhao, Z., et al., Fibroatheroma Morphological Features of Borderline Coronary Lesion Plaques on Stable Angina Pectoris Patients. Enliven: Clin Cardiol Res, 2015. 2(1): p. 002.

39. Hong, Y.J., et al., Plaque components at coronary sites with focal spasm in patients with variant angina: Virtual histology-intravascular ultrasound analysis. International journal of cardiology, 2010. 144(3): p. 367-372.

40. Wang, R., et al. Multi features combination for automated zooplankton classification. in OCEANS -Shanghai. 2016. IEEE.

41. Duda, R.O., P.E. Hart, and D.G. Stork, Pattern classification. 2012: John Wiley \& Sons.

42. Kennedy, R.L., et al., Solving Data Mining Problems Through Pattern Recognition. Prentice-Hall, PTR, Upper Saddle River, NJ, 1998: p. 11-16 (Chapter 9).

43. Falasconi, M., et al., A stability based validity method for fuzzy clustering. Pattern Recognition, 2010. 43(4): p. 1292-1305.

44. Chavan, N.V., B. Jadhav, and P. Patil, Detection and classification of brain tumors. International Journal of Computer Applications, 2015. 112(8): p. 48-53.

45. Downe, R.W., Predictive analysis of coronary plaque morphology and composition on a one year timescale. University of Iowa, Iowa, 2013.

46. Hong, Y.J., et al., Impact of plaque components on no-reflow phenomenon after stent deployment in patients with acute coronary syndrome: a virtual histology-intravascular ultrasound analysis. European heart journal, 2011. 32(16): p. 2059-2066. 